\title{
BI-ISOTHERMAL SYSTEMS
}

\section{EDWARD KASNER AND JOHN DECICCO}

1. Introduction. A pair of functions of two complex variables with nonvanishing jacobian induces a correspondence between the points of a real (or complex) four-dimensional euclidean space $R_{4}$. The infinite group $G$ of all such correspondences is not the conformal group of $R_{4}$, which is merely the inversive group of fifteen parameters. In 1908 , the senior author termed $G$ the pseudo-conformal group. This is now standard terminology. (See papers by Cartan, Carathéodory, and S. Bergmann.) It will be recalled that Poincaré, in his fundamental Palermo memoir of 1907 , tentatively called $G$ the regular group.

In 1908, Kasner showed that the pseudo-conformal group $G$ is characterized by the preservation of the pseudo-angle between any curve and a hypersurface at their common point of intersection. ${ }^{1}$ This is a proper generalization of the well known result that the group of functions of a single complex variable is identical with the (direct) conformal group of the plane.

In $R_{4}$, there is a class of surfaces which is transformed into itself under the infinite pseudo-conformal group $G$ such that the induced correspondence between any corresponding pair of such surfaces is (direct) conformal. Any such surface is said to be a conformal surface. The term analytic surface is also in use.

Upon projecting orthogonally (by means of absolutely perpendicular planes) a conformal surface upon a pair of selected coordinate planes, the three induced correspondences (surface on each of the two planes, and plane on plane) are each conformal. Thus any conformal surface may be defined by means of a conformality between the selected pair of coordinate planes.

We shall say that a bi-isothermal system of $\infty^{3}$ curves in $R_{4}$ is any system which is pseudo-conformally equivalent to a parallel pencil of $\infty^{3}$ straight lines in $R_{4}$. Any such system consists of $\infty^{2}$ isothermal families of $\infty^{1}$ curves, each such family lying upon a conformal surface.

We define a bi-isothermal system of $\infty^{1}$ hypersurfaces in $R_{4}$ as any system which is pseudo-conformally equivalent to a parallel pencil of $\infty^{1}$ hyperplanes in $R_{4}$. Any bi-isothermal system of hypersurfaces may be defined by placing a biharmonic function equal to an arbi-

Presented to the Society, December 27, 1942; received by the editors July 6, 1944.

1 Kasner, Conformality in connection with functions of two complex variables, Trans. Amer. Math. Soc. vol. 48 (1940) pp. 50-62. 
trary constant. In that case, the constant may be called the $b i$-isothermal parameter. Of course, it is seen that, in general, a bi-isothermal system of $\infty^{1}$ hypersurfaces may be defined by placing a function of a biharmonic function equal to an arbitrary constant.

We find the following theorems on bi-isothermals fundamental in the geometry of functions of two complex variables.

1. The pseudo-angle between any bi-isothermal system of hypersurfaces and any bi-isothermal system of curves is a biharmonic function. This may be considered to be an extension of a theorem of Lie concerning isothermal systems in the plane. (See also a generalization to surfaces to appear in Proc. Nat. Acad. Sci. U.S.A., February, 1945.)

2. Any bi-isothermal system of hypersurfaces is intersected by a conformal surface in an isothermal system of curves. 3. If a surface is intersected by every bi-isothermal system of hypersurfaces in an isothermal system of curves, then the surface is conformal. 4. If a given system of $\infty^{1}$ hypersurfaces is intersected by every conformal surface in an isothermal system of curves, then the given system of $\infty^{1}$ hypersurfaces must be bi-isothermal. ${ }^{2}$

2. Minimal coordinates. Let $\left(x_{1}, x_{2}^{2} ; y_{1}, y_{2}\right)$ define cartesian coordinates in the euclidean space $R_{4}$. We shall find it convenient to introduce the minimal coordinates defined by

$$
\begin{array}{ll}
u_{1}=x_{1}+i y_{1}, & v_{1}=x_{1}-i y_{1}, \\
u_{2}=x_{2}+i y_{2}, & v_{2}=x_{2}-i y_{2} .
\end{array}
$$

It is noted that the relations between the partial derivative operators in minimal and cartesian coordinates are

$$
\begin{array}{lll}
\frac{\partial}{\partial u_{1}}=\frac{1}{2}\left(\frac{\partial}{\partial x_{1}}-i \frac{\partial}{\partial y_{1}}\right), & \frac{\partial}{\partial v_{1}}=\frac{1}{2}\left(\frac{\partial}{\partial x_{1}}+i \frac{\partial}{\partial y_{1}}\right), \\
\frac{\partial}{\partial u_{2}}=\frac{1}{2}\left(\frac{\partial}{\partial x_{2}}-i \frac{\partial}{\partial y_{2}}\right), & \frac{\partial}{\partial v_{2}}=\frac{1}{2}\left(\frac{\partial}{\partial x_{2}}+i \frac{\partial}{\partial y_{2}}\right) .
\end{array}
$$

The operators $\partial / \partial u_{1}$ and $\partial / \partial u_{2}$ are called the mean derivatives and the operators $\partial / \partial v_{1}$ and $\partial / \partial v_{2}$ are termed the phase derivatives. ${ }^{3}$

2 These theorems may be considered to be extensions of various theorems given in the paper of Kasner, Biharmonic functions and certain generalizations, Amer. J. Math. vol. 58 (1936) pp. 377-390.

${ }^{3}$ Kasner, The second derivative of a polygenic function, Trans. Amer. Math. Soc. vol. 30 (1928) pp. 803-818. Also Kasner and DeCicco, The derivative circular congruence-representation of a polygenic function, Amer. J. Math. vol. 61 (1939) pp. 995-1003. Also a forthcoming paper in Scripta Mathematica, 1945. 
In minimal coordinates, the square of the linear element $d s$ is

$$
d s^{2}=d u_{1} d v_{1}+d u_{2} d v_{2} .
$$

The cosine of the angle $\theta$ between any two curve elements through a common point is

$$
\cos \theta=\frac{d u_{1}^{(1)} d v_{1}^{(2)}+d u_{1}^{(2)} d v_{1}^{(1)}+d u_{2}^{(1)} d v_{2}^{(2)}+d u_{2}^{(2)} d v_{2}^{(1)}}{2\left[\left(d u_{1}^{(1)} d v_{1}^{(1)}+d u_{2}^{(1)} d v_{2}^{(1)}\right)\left(d u_{1}^{(2)} d v_{1}^{(2)}+d u_{2}^{(2)} d v_{2}^{(2)}\right)\right]^{1 / 2}} .
$$

3. The infinite continuous pseudo-conformal group $G$. This is defined by

$$
\begin{array}{ll}
U_{1}=U_{1}\left(u_{1}, u_{2}\right), & V_{1}=V_{1}\left(v_{1}, v_{2}\right), \\
U_{2}=U_{2}\left(u_{1}, u_{2}\right), & V_{2}=V_{2}\left(v_{1}, v_{2}\right),
\end{array}
$$

where the jacobians $\partial\left(U_{1}, U_{2}\right) / \partial\left(u_{1}, u_{2}\right)$ and $\partial\left(V_{1}, V_{2}\right) / \partial\left(v_{1}, v_{2}\right)$ are each not zero.

We have already found all the possible differential invariants of the first order of this infinite continuous pseudo-conformal group $G .{ }^{4}$

4. Kasner's pseudo-angle. A hypersurface $S_{3}: F\left(u_{1}, u_{2} ; v_{1}, v_{2}\right)=0$ and a curve $C: u_{1}=u_{1}(t), u_{2}=u_{2}(t) ; v_{1}=v_{1}(t), v_{2}=v_{2}(t)$ possess the fundamental differential invariant of first order

$$
\theta=\frac{1}{2 i} \log \left[-\frac{F_{v_{1}} d v_{1}+F_{v_{2}} d v_{2}}{F_{u_{1}} d u_{1}+F_{u_{2}} d u_{2}}\right] .
$$

This represents the actual angle $\theta$ between the given curve $C$ and the curve of intersection $C^{\prime}$ between the hypersurface $S_{3}$ and the unique conformal surface determined by the curve $C$.

5. Bi-isothermal systems of $\infty^{3}$ curves. A system of $\infty^{3}$ curves is $b i$-isothermal if it is pseudo-conformally equivalent to a parallel pencil of straight lines in $R_{4}$. By means of (5), it is seen that any bi-isothermal system of $\infty^{3}$ curves may be given by equations of the forms

$$
\begin{gathered}
\lambda\left(u_{1}, u_{2}\right)=\text { const., } \quad \mu\left(v_{1}, v_{2}\right)=\text { const., } \\
\nu\left(u_{1}, u_{2}\right)+\omega\left(v_{1}, v_{2}\right)=\text { const. }
\end{gathered}
$$

It therefore follows that any such system of $\infty^{3}$ curves may be defined by a system of three differential equations of the forms

$$
\begin{gathered}
d u_{2} / d u_{1}=\alpha\left(u_{1}, u_{2}\right), \quad d v_{2} / d v_{1}=\beta\left(v_{1}, v_{2}\right), \\
d v_{1} / d u_{1}=\gamma\left(v_{1}, v_{2}\right) / \delta\left(u_{1}, u_{2}\right) .
\end{gathered}
$$

4 Kasner and DeCicco, Pseudo-conformal geometry: Functions of two complex variables, Bull. Amer. Math. Soc. vol. 48 (1942) pp. 317-328. 
Conversely, the $\infty^{3}$ integral curves of any system of differential equations reducible to the form (8) must be a bi-isothermal system.

Since any conformal surface is given by equations of the form $l\left(u_{1}, u_{2}\right)=0, m\left(v_{1}, v_{2}\right)=0$, it follows by $(7)$ that any bi-isothermal system of $\infty^{3}$ curves consists of $\infty^{2}$ isothermal families of $\infty^{1}$ curves, each such family lying on a conformal surface.

6. Bi-isothermal systems of $\infty^{1}$ hypersurfaces. Any system of $\infty^{1}$ hypersurfaces which is pseudo-conformally equivalent to a parallel pencil of $\infty^{1}$ hyperplanes is said to be bi-isothermal. Clearly the system

$$
f\left(u_{1}, u_{2}\right)+g\left(v_{1}, v_{2}\right)=\text { const. }
$$

is bi-isothermal. In this form, the constant is said to be a bi-isothermal parameter.

Conversely let $F\left(u_{1}, u_{2} ; v_{1}, v_{2}\right)=$ const. define a bi-isothermal system of $\infty^{1}$ hypersurfaces. There must exist a function $\phi(F)$ which is a biharmonic function of $\left(u_{1}, u_{2} ; v_{1}, v_{2}\right)$. Therefore, in consequence of the fundamental Poincaré conditions, we find

$$
\Delta=\frac{F_{u_{1} v_{1}}}{F_{u_{1}} F_{v_{1}}}=\frac{F_{u_{2} v_{2}}}{F_{u_{2}} F_{v_{2}}}=\frac{F_{u_{1} v_{2}}}{F_{u_{1}} F_{v_{2}}}=\frac{F_{u_{2} v_{1}}}{F_{u_{2}} F_{v_{1}}}
$$

must be a function of $F$ only. Thus $\Delta$ must satisfy the additional third-order differential equations

$$
\frac{\Delta_{u_{1}}}{F_{u_{1}}}=\frac{\Delta_{u_{2}}}{F_{u_{2}}}=\frac{\Delta_{v_{1}}}{F_{v_{1}}}=\frac{\Delta_{v_{2}}}{F_{v_{2}}} .
$$

Therefore, we have proved the following result.

THEOREM 1. The necessary and sufficient conditions that $F\left(u_{1}, u_{2}\right.$; $\left.v_{1}, v_{2}\right)=$ const. define a bi-isothermal system of $\infty^{1}$ hypersurfaces is that $F$ satisfy the system of six partial differential equations of third order, given by (10) and (11).

Upon substituting (8) and (9) into (6), we discover the following proposition.

THEOREM 2. The pseudo-angle between any bi-isothermal system of $\infty^{1}$ hypersurfaces and any bi-isothermal system of $\infty^{3}$ curves is a biharmonic function of $\left(u_{1}, u_{2} ; v_{1}, v_{2}\right)$, that is, $\theta$ is given by an equation of the form $\theta=h\left(u_{1}, u_{2}\right)+k\left(v_{1}, v_{2}\right)$.

In the next and final section, we shall give certain theorems which 
may be considered to be generalizations of certain theorems of Kasner which characterize biharmonic functions.

7. Bi-isothermal systems of $\infty^{1}$ hypersurfaces and conformal surfaces. A conformal surface $S_{2}$ induces a conformal correspondence between the $\left(x_{1}, y_{1}\right)$ and the $\left(x_{2}, y_{2}\right)$ coordinate planes. Thus $S_{2}$ is defined by the equations $u_{2}=u_{2}\left(u_{1}\right), v_{2}=v_{2}\left(v_{1}\right)$. By the induced conformal correspondences, it follows that an isothermal system on the $\left(x_{1}, y_{1}\right)$ coordinate plane corresponds to an isothermal system on the $\left(x_{2}, y_{2}\right)$ plane, and also to an isothermal system on the given conformal surface $S_{2}$.

THEOREM 3. Any bi-isothermal system of $\infty^{1}$ hypersurfaces is intersected by a conformal surface in an isothermal system.

This result is obtained by substituting the equations of a conformal surface into the equation (9), defining a bi-isothermal system of hypersurfaces.

THEOREM 4. If a surface is intersected by every bi-isothermal system of $\infty^{1}$ hypersurfaces in an isothermal system of curves, then the surface is a conformal surface.

Any surface $S_{2}$ may be given by the equations $u_{2}=u_{2}\left(u_{1}, v_{1}\right)$, $v_{2}=v_{2}\left(u_{1}, v_{1}\right)$. Upon substituting this into the equation (9) defining any bi-isothermal system, the resulting system of $\infty^{1}$ curves must be isothermal. Hence

$$
\frac{\partial^{2}}{\partial u_{1} \partial v_{1}} \log \left[\frac{f_{u_{1}}+f_{u_{2}} \partial u_{2} / \partial u_{1}+g_{v_{2}} \partial v_{2} / \partial u_{1}}{g_{v_{1}}+g_{v_{2}} \partial v_{2} / \partial v_{1}+f_{u_{2}} \partial u_{2} / \partial v_{1}}\right]=0 .
$$

This must be an identity for all values of the partial derivatives of $f$ and $g$.

The above equation involves partial derivatives of the third order in $f$ and $g$. Upon setting the coefficients of $f_{u_{1} u_{1} u_{2}}$ and $g_{v_{1} v_{1} v_{2}}$ equal to zero, we discover that $\partial u_{2} / \partial v_{1}=0$ and $\partial v_{2} / \partial u_{1}=0$. This proves that the surface $S_{2}$ is conformal. Theorem 4 is completely proved.

THEOREM 5. If a given system of $\infty^{1}$ hypersurfaces is intersected by every conformal surface in an isothermal family of curves, then the system of $\infty^{1}$ hypersurfaces is bi-isothermal.

Let the given system of $\infty^{1}$ hypersurfaces be $F\left(u_{1}, u_{2} ; v_{1}, v_{2}\right)=$ const. Substituting the equations of any conformal surface $u_{2}=u_{2}\left(u_{1}\right)$, $v_{2}=v_{2}\left(v_{1}\right)$ into it, the resulting family must be isothermal. Hence we have 


$$
\frac{\partial^{2}}{\partial u_{1} \partial v_{1}} \log \left[\frac{F_{u_{1}}+F_{u_{2}} d u_{2} / d u_{1}}{F_{v_{1}}+F_{v_{2}} d v_{2} / d v_{1}}\right]=0 .
$$

This is a differential equation of the second order in the total derivatives of $u_{2}=u_{2}\left(u_{1}\right), v_{2}=v_{2}\left(v_{1}\right)$ which must be identically zero. Upon setting the coefficients of $d^{2} u_{2} / d u_{1}^{2}$ and $d^{2} v_{2} / d v_{1}^{2}$ equal to zero, we find

$$
\begin{aligned}
& \left(F_{u_{1}} F_{u_{2} v_{1}}-F_{u_{2}} F_{u_{1} v_{1}}\right)+\left(F_{u_{1}} F_{u_{2} v_{2}}-F_{u_{2}} F_{u_{1} v_{2}}\right) d v_{2} / d v_{1}=0, \\
& \left(F_{v_{1}} F_{u_{1} v_{2}}-F_{v_{2}} F_{u_{1} v_{1}}\right)+\left(F_{v_{1}} F_{u_{2} v_{2}}-F_{v_{2}} F_{u_{2} v_{1}}\right) d u_{2} / d u_{1}=0 .
\end{aligned}
$$

These identities yield the equations (10).

Substituting these into the equation (13), we find

$$
\frac{\partial}{\partial u_{1}}\left[F_{v_{1}}+F_{v_{2}} \frac{d v_{2}}{d v_{1}}\right] \Delta=\frac{\partial}{\partial v_{1}}\left[F_{u_{1}}+F_{u_{2}} \frac{d u_{2}}{d u_{1}}\right] \Delta .
$$

Upon setting the various coefficients of $d u_{2} / d u_{1}$ and $d v_{2} / d v_{1}$ of the above equation equal to zero, we obtain the equations (11).

Thus the hypothesis of Theorem 5 has led us to the conditions (10) and (11) for a bi-isothermal system of $\infty^{1}$ hypersurfaces. This completes our proof.

Columbia University aNd

ILLINOIS INSTITUTE OF TECHNOLOGY 